\title{
Liquid drop in a cone - line tension effects
}

\author{
G P Kubalski and M Napiórkowski \\ Institute of Theoretical Physics, Warsaw University, Hoża 69, 00-681 Warsaw, Poland
}

\begin{abstract}
The shape of a liquid drop placed in a cone is analyzed macroscopically. Depending on the values of the cone opening angle, the Young angle and the line tension four different interfacial configurations may be realized. The phase diagram in these variables is constructed and discussed; it contains both the first- and the secondorder transition lines. In particular, the tricritical point is found and the value of the critical exponent characterizing the behaviour of the system along the line of the first-order transitions in the neighbourhood of this point is determined.
\end{abstract}

PACS numbers: 68.55.Jk, 68.35.Rh, 68.45.Gd 


\section{Introduction}

The role of line and surface tensions in determining the morphology of interfaces is well known [1]. For example, the values of the contact angles formed by an interface and a substrate depend on both the line and the surface tensions. In the simplest case of a drop sessile on a flat substrate the influence of line tension is described by the modified Young equation [4, [7]. Under special circumstances one can even expect a competition between the interfacial tension and the line tension effects. This competition originates from the fact that while the interfacial tension is positive [8] and tends to decrease the interfacial area the negative values of the line tension [9] support the increase of the three-phase contact line. This competition between the line and the surface tension effects may result in forming different interfacial configurations and transitions between them. For example, Widom [10] in his analysis of a sessile drop on flat substrate found a line of first-order phase transitions parameterized by the line tension.

In this paper we analyze the equilibrium shapes of a non-volatile liquid drop placed in a cone by taking into account both the surface tension and the line tension effects. The system and our methods of macroscopic analysis are described in Section 2. In Section 3 we find the equilibrium configurations of the liquid which are parameterized by the values of the cone opening angle, the Young angle - which itself depends on the values of the interfacial and surface tensions - and the line tension. This section is divided into two parts. In the first part the case of zero line tension is considered analytically; in the second part we solve numerically the non-zero line tension case. The phase diagram found in this section is our main result. The last section contains the conclusions and remarks.

\section{Macroscopic analysis}

We consider a given amount of liquid placed on a nondeformable solid substrate which has the shape of a cone with the opening angle $2 \psi, \psi<\pi / 2$. The bulk thermodynamic conditions, say the temperature and the pressure are chosen such that the liquid is in equilibrium with its vapour. The liquid can form one or more separate droplets, see figure 1. The physical parameters used in our analysis are the liquid-vapour, substrateliquid, and substrate-vapour surface tensions denoted as $\sigma_{l v}, \sigma_{s l}, \sigma_{s v}$, respectively; the line tension accompanying the substrate-liquid-vapour contact line is denoted as $\eta_{s l v}$. The gravity effects are neglected; for the discussion of their role see [10].

The configurations of the liquid are uniquely determined by the shapes of the liquidvapour interfaces. The shape of the $i$-th interface is described by the function $f_{i}$. Thus the set $\left\{f_{i}\right\}_{i=1}^{n}$ describes the liquid configuration with $n$ liquid-vapour interfaces num-

bered from the top to the bottom of the cone. Our analysis is restricted to configurations with cylindrical symmetry and interfaces which are smooth at the cone axis. In consequence all functions $f_{i}$ depend on only one variable - the distance $\rho$ from the cone axis. 


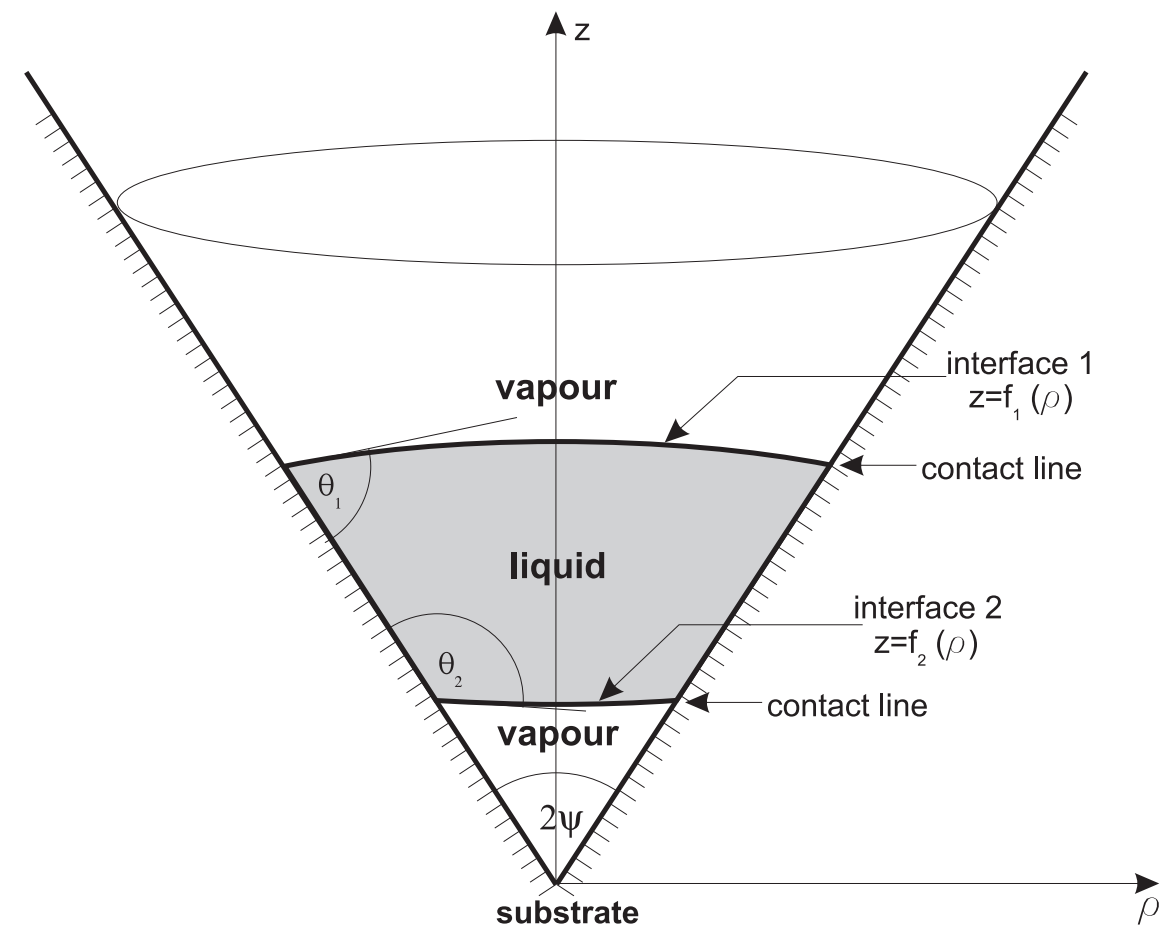

Figure 1. The schematic plot of the system. As an example the configuration with two interfaces is shown. The z-axis coincides with the axis of the cone.

We start by constructing the macroscopic constrained free energy which is a functional of interfacial configurations. Then we find the equilibrium configurations which correspond to the minima of this functional. Actually we consider the excess free energy with respect to configuration in which the cone is filled the the vapour only. This excess free energy is denoted by $\mathcal{F}\left[\left\{f_{i}\right\}_{i=1}^{n}\right]$ and has the following form

$$
\mathcal{F}=A_{l v} \sigma_{l v}+A_{s l}\left(\sigma_{s l}-\sigma_{s v}\right)+L \eta_{s l v},
$$

where $A_{l v}, A_{s l}$ denote the area of the liquid-vapour and substrate-liquid interfaces, respectively and $L$ is the length of the three-phase contact line. After substituting the expression for $A_{l v}, A_{s l}$, and $L$ in terms of $\left\{f_{i}\right\}_{i=1}^{n}$ into equation (1) one obtains

$$
\begin{aligned}
\mathcal{F}\left[\left\{f_{i}\right\}_{i=1}^{n}\right]= & 2 \pi \sigma_{l v} \int_{0}^{\infty}\left(\sum _ { i = 1 } ^ { n } \theta ( f _ { i } ( \rho ) - \rho \operatorname { c t g } \psi ) \left(\sqrt{1+\left(\frac{d f_{i}}{d \rho}\right)^{2}}+\right.\right. \\
& \left.\left.\frac{1}{\sin \psi}\left((-1)^{i} \frac{\sigma_{s v}-\sigma_{s l}}{\sigma_{l v}}+\frac{\eta_{s l v} \sin \psi}{\sigma_{l v} \rho}\right)\right)\right) \rho d \rho
\end{aligned}
$$

$\theta$ denotes the Heaviside function. We look for the minima of the above functional under the constraint of fixed volume $V$ of the liquid. Thus the functional to be minimized takes the form

$$
\mathcal{F}^{*}\left[\left\{f_{i}\right\}_{i=1}^{n}\right]=\mathcal{F}\left[\left\{f_{i}\right\}_{i=1}^{n}\right]+\lambda V\left[\left\{f_{i}\right\}_{i=1}^{n}\right],
$$


Liquid drop in a cone - line tension effects

where $\lambda$ is the Lagrange multiplier and

$$
V=2 \pi \int_{0}^{\infty}\left(\sum_{i=1}^{n}(-1)^{i+1}\left(\theta\left(f_{i}(\rho)-\rho \operatorname{ctg} \psi\right)\left(f_{i}(\rho)-\rho \operatorname{ctg} \psi\right)\right) \rho d \rho .\right.
$$

Accordingly the equilibrium shape of the $i$-th liquid-vapour interface fulfills the following differential equation

$$
\left(1+\left(\frac{d f_{i}}{d \rho}\right)^{2}\right)^{-3 / 2}\left(\frac{d^{2} f_{i}}{d \rho^{2}} \rho+\left(1+\left(\frac{d f_{i}}{d \rho}\right)^{2}\right) \frac{d f_{i}}{d \rho}\right)=(-1)^{i+1} \frac{\lambda}{\sigma_{l v}} \rho
$$

supplemented by the boundary conditions

$$
f\left(\rho_{i}\right)=\rho_{i} \operatorname{ctg} \psi,
$$

where $\rho_{i}$ denotes the distance from the cone axis at which the $i$-th interface touches the substrate, and

$$
\left(1+\left(\frac{d f_{i}}{d \rho}\right)^{2}\right)^{-1 / 2}\left(1+\operatorname{ctg} \psi \frac{d f_{i}}{d \rho}\right)=(-1)^{i+1} \frac{1}{\sin \psi} \frac{\sigma_{s v}-\sigma_{s l}}{\sigma_{l v}}-\frac{\eta_{s l v}}{\sigma_{l v} \rho_{i}}
$$

where all the derivatives are calculated at $\rho=\rho_{i}$. Equation (7) is equivalent to the statement that the contact angle $\theta_{i}$ of $i$-th interface (defined as the angle between this interface and the substrate measured across the liquid, see figure 1) fulfills the following form of the modified Young equation for the cone [4, 7]

$$
\cos \theta_{i}=\cos \theta_{0}+(-1)^{i+1} \frac{\eta_{s l v}}{\sigma_{l v} \rho_{i}}
$$

$\theta_{0}$ denotes the Young angle, i.e. the contact angle of the liquid drop on a flat substrate and in the case of zero line tension

$$
\cos \theta_{0}=\frac{\sigma_{s v}-\sigma_{s l}}{\sigma_{l v}}
$$

The solution of (5) which is smooth on the cone axis and satisfies the boundary condition (6) has the form

$$
f_{i}(\rho)=\rho_{i} \operatorname{ctg} \psi+(-1)^{i} \operatorname{sgn}(\lambda)\left(\sqrt{\left(\frac{2 \sigma_{l v}}{\lambda}\right)^{2}-\rho^{2}}-\sqrt{\left(\frac{2 \sigma_{l v}}{\lambda}\right)^{2}-\rho_{i}^{2}}\right) .
$$

Thus each liquid-vapour interface forms a part of a sphere with radius $R=2 \sigma_{l v} / \lambda$. It can be determined from the fixed volume constraint (四)

$$
\begin{aligned}
R=\left(\frac{3 V}{\pi}\right)^{1 / 3} & \left(\sum _ { i = 1 } ^ { n } \left(\cos ^{2}\left(\theta_{i}+(-1)^{i+1} \psi\right)(-1)^{i+1} \frac{\cos \theta_{i}}{\sin \psi}+\right.\right. \\
& \left.\left.-2\left(1-\sin \left(\theta_{i}+(-1)^{i+1} \psi\right)\right)\right)\right)^{-1 / 3} .
\end{aligned}
$$

The location of the three-phase contact line of the $i$-th interface is given by

$$
\rho_{i}=R \cos \left(\theta_{i}+(-1)^{i+1} \psi\right) .
$$

After introducing the dimensionless quantities

$$
\overline{\mathcal{F}}=\frac{\mathcal{F}}{2 \pi\left(\frac{3 V}{\pi}\right)^{2 / 3} \sigma_{l v}},
$$


Liquid drop in a cone - line tension effects

$$
\begin{aligned}
& \bar{R}=\frac{R}{\left(\frac{3 V}{\pi}\right)^{1 / 3}}, \\
& \bar{\eta}=\frac{\eta_{s l v}}{\sigma_{l v}\left(\frac{3 V}{\pi}\right)^{1 / 3}} .
\end{aligned}
$$

the free energy of a given drop configuration can be expressed in the following form

$$
\begin{aligned}
\overline{\mathcal{F}}_{n}=\bar{R}^{2} \sum_{i=1}^{n}\left(1-\sin \left(\theta_{i}+(-1)^{i+1} \psi\right)+\right. \\
\left.\quad(-1)^{i} \frac{\cos \theta_{0}}{2 \sin \psi} \cos ^{2}\left(\theta_{i}+(-1)^{i+1} \psi\right)+\frac{\bar{\eta}}{\bar{R}} \cos \left(\theta_{i}+(-1)^{i+1} \psi\right)\right),
\end{aligned}
$$

where the subscript $n$ in $\bar{F}_{n}$ refers to the case with exactly $n$ - interfaces present in the system. The modified Young equation takes the form

$$
\cos \theta_{i}-\cos \theta_{0}+\frac{\bar{\eta}}{\bar{R}} \frac{(-1)^{i+1} \sin \psi}{\cos \left(\theta_{i}+(-1)^{i+1} \psi\right)}=0 .
$$

It can be checked that equations (16) and (17) remain valid for all contact angles, so our primary assumptions that $\vartheta_{i}<\pi-\psi$ may be discarded.

In the above formulae - in addition to the contact angles $\theta_{i}$ - only the parameters $\theta_{0}, \bar{\eta}$ and $\psi$ appear. Thus from now on this set of three variables will be used to parameterize the equilibrium properties of the system. Note that the volume $V$ enters the rhs of (16) only via the expression for $\bar{\eta}$ where it rescales the line tension.

\section{Equilibrium drop configurations}

\subsection{Zero line tension}

In this subsection we put $\bar{\eta}=0$. In this case the solution of (17) takes the form

$$
\theta_{i}=\theta_{0}
$$

which means that only configurations with one and two interfaces are allowed. These configurations we will be called the blob and the bridge configuration, respectively [11, 12. The corresponding free energies take the following form

$$
\bar{F}_{1}=2^{-2 / 3}\left(\left(1-\sin \left(\theta_{0}+\psi\right)\right)-\frac{\cos \theta_{0}}{2 \sin \psi} \cos ^{2}\left(\theta_{0}+\psi\right)\right)^{1 / 3}
$$

(blob configuration) and

$$
\begin{aligned}
\bar{F}_{2}=2^{-2 / 3}((1 & \left.-\sin \left(\theta_{0}+\psi\right)\right)+\left(1-\sin \left(\theta_{0}-\psi\right)\right)+ \\
& \left.-\frac{\cos \theta_{0}}{2 \sin \psi}\left(\cos ^{2}\left(\theta_{0}+\psi\right)-\cos ^{2}\left(\theta_{0}-\psi\right)\right)\right)^{1 / 3}
\end{aligned}
$$

(bridge configuration). Comparing the above free energies leads to the conclusion that for $\theta_{0} \leq \pi / 2+\psi$ the equilibrium configuration has the form of a blob while for $\theta_{0}>\pi / 2+\psi$ it takes the form of a bridge. According to (12) the transition between the blob and the bridge configurations corresponds to the situation in which the bottom 


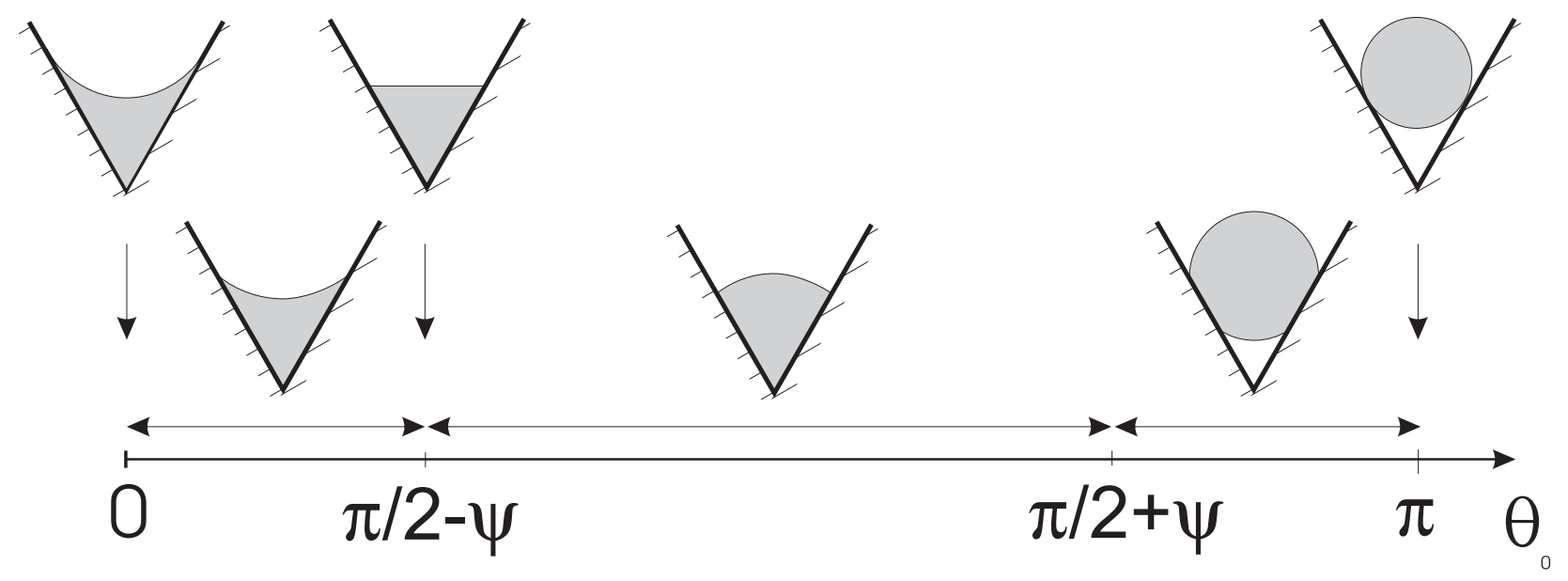

Figure 2. The phase diagram in the case of zero line tension. The point $\theta_{0}=$ $\psi+\pi / 2$ corresponds to the continuous transition between the blob and the bridge configurations.

interface of the bridge reaches the vertex of the cone. This transition is continuous. This results is similar to the one for adsorption in a wedge [12]. Figure 2 shows the relevant phase diagram.

\subsection{Non-zero line tension}

For $\eta \neq 0$ the analysis of (17) shows that - similarly to the case of zero line tension - only configurations with one and two interfaces are admitted. This case, contrary to the previous one can be treated only numerically. One method of finding the equilibrium values of the contact angles is to solve numerically (17) and then to calculate the corresponding values of the free energy in order to find the equilibrium solution. However, it turns out that the direct numerical minimization of the constrained free energy is more efficient procedure in determining the equilibrium configurations and we follow this method [13].

The equilibrium configuration of the drop - for given set of $\theta_{0}, \bar{\eta}$, and $\psi$ values corresponds to the global minimum of the free energy. In practice one has to find all the

minima of (16) corresponding to the blob $(n=1)$ and the bridge $(n=2)$ configurations including also the minima localized on the borders of contact angle domain, i.e. $\theta_{1}$ or $\theta_{2}$ equal to 0 or $\pi$, and then to compare the values of the corresponding free energy. Table 1 introduces the terminology used in describing the phase diagram.

Figure 3 shows schematically the generic phase diagram; figure 4 presents the actual phase diagrams corresponding to particular values of the cone opening angle.

Before discussing the phase diagram it is worthwhile to recall that for given value of the cone opening angle $\psi$ the phase diagram is constructed in function of the Young 
Table 1. Configurations of liquid in a cone.

\begin{tabular}{lccc}
\hline configuration & number of interfaces & $\theta_{1}$ & $\theta_{2}$ \\
\hline blob & 1 & $\neq 0, \neq \pi$ & - \\
wetting & 1 & $=0$ & - \\
quasi-drying & 1 & $=\pi$ & - \\
bridge & 2 & $\neq 0, \neq \pi$ & $\neq 0, \neq \pi$ \\
lower drying & 2 & $\neq 0, \neq \pi$ & $=\pi$ \\
upper drying & 2 & $=\pi$ & $\neq 0, \neq \pi$ \\
drying & 2 & $=\pi$ & $=\pi$ \\
\hline
\end{tabular}

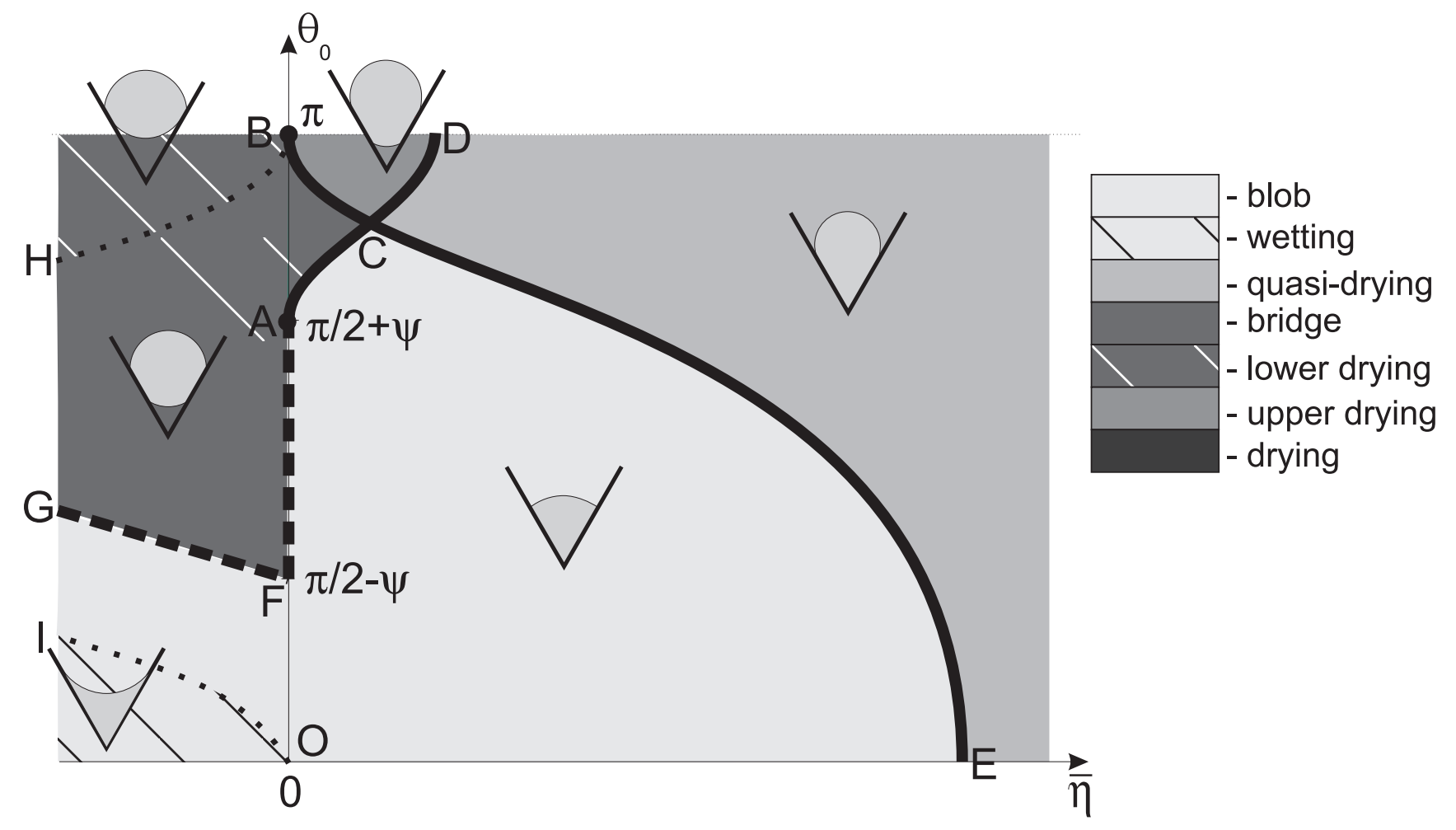

Figure 3. The generic phase diagram. Different shades of gray correspond to different phases which are schematically depicted in the insets. The continuous lines B-E and A-D correspond to the first-order phase transitions; the broken line A-F-G corresponds to the continuous transition. Point A denotes the tricritical point. The dotted line IO forms the border of the region in which the contact angle $\theta_{1}=0$; the dotted line HB forms the border of the region in which the contact angle $\theta_{2}=\pi$. 
a)

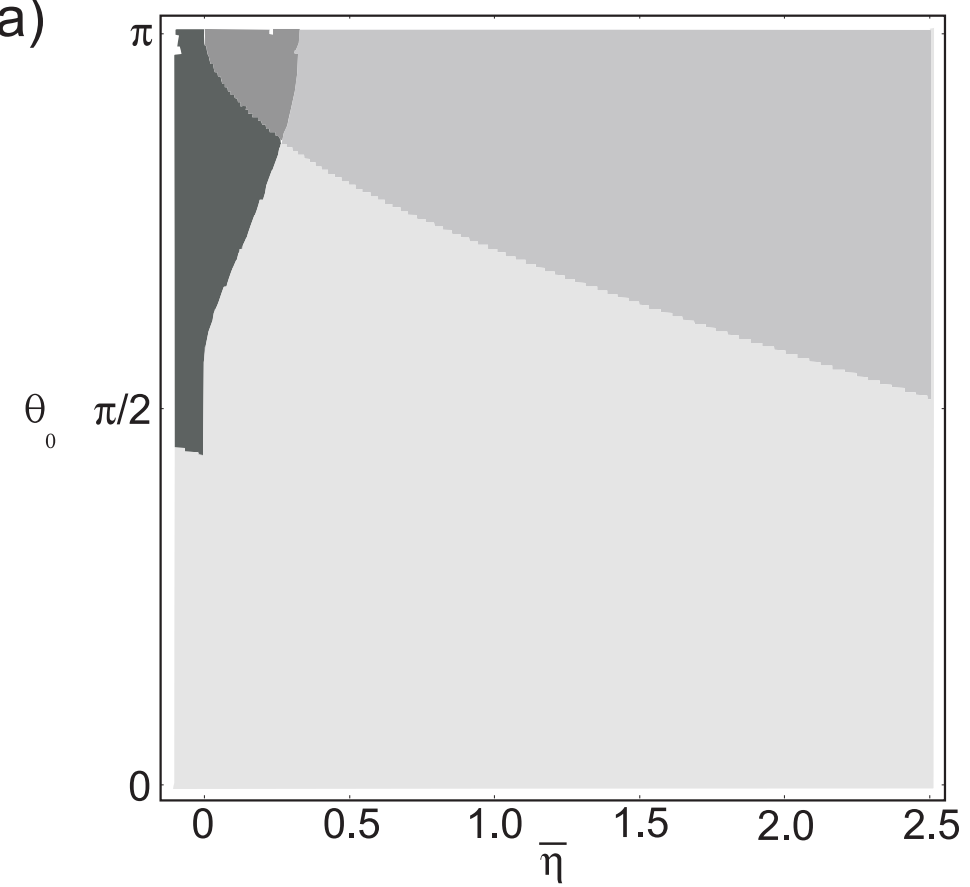

b)

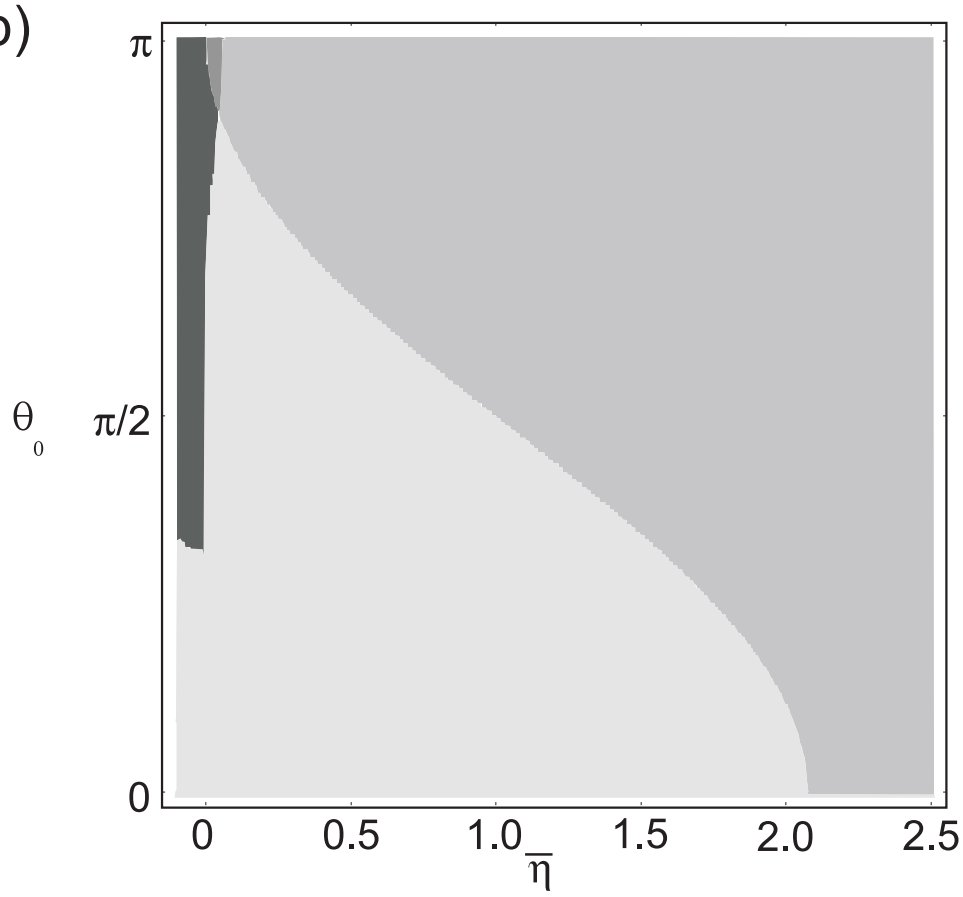

Figure 4. The phase diagrams for two particular choices of the cone opening angle: a) $\psi=\pi / 16$, b) $\psi=3 \pi / 16$. The shades of gray correspond to those used in figure 3 . Note that the regions corresponding to the wetting and lower drying configurations are not marked on this figure. 
angle $\theta_{0}$, eq.(8) and the dimensionless line tension $\bar{\eta}$, Eq.(12c) which is inversely proportional to the cubic root of the liquid volume $V$. This means that substantial influence of the line tension can be observed only for drops of sufficiently small volume. If one takes the typical value of line tension to be $10^{-8} \mathrm{~N}$ [14], the typical value of liquid-vapour surface tension to be $10^{-2} \mathrm{~N} / \mathrm{m}$ [15], and the size of the liquid drop to be of micrometer size then the dimensionless line tension is of order unity. For much bigger drops the effects of line tension will be negligible.

The rich structure of the phase diagram results from the competition between the surface tension and the line tension. Depending on the values of parameters $\theta_{0}$ and $\bar{\eta}$ the system can be found in one of four different phases. They are distinguished by the number of interfaces present in the system (one or two) and the value of the contact angle $\theta_{1}$ (corresponding to the upper interface) being equal to $\pi$ or being different from $\pi$, see also Table 1 . These four phases are denoted on figure 3 with the help of different shades of gray and are separated from each other by thick continuous or thick broken lines depending on the order of the transition. To make the phase diagram more transparent each phase is schematically marked by the corresponding liquid configuration. Additionally, within two of the above phases one can distinguish quantitatively different regions. The lower left part of the phase diagram contains region corresponding to configuration with only one interface and zero contact angle; we call it the wetting configuration. The dotted line I-O marks the boundary of this region; upon crossing this line the contact angle ceases to be equal to 0 . The upper left part of the phase diagram corresponds to configuration with two interfaces and the contact angle $\theta_{2}$ corresponding to the lower interface being equal to $\pi$. Again the dotted line $\mathrm{H}-\mathrm{B}$ marks the boundary of this region; upon crossing it the contact angle $\theta_{2}$ ceases to be equal to $\pi$.

The line B-E is the first-order transition line between configurations distinguished by the value of the contact angle $\theta_{1}$ being equal to $\pi$ and different from $\pi$. Note that the segment C-E corresponds to the first-order transition between phases in which only one interface is present while the segment $\mathrm{B}-\mathrm{C}$ corresponds to the first-order transition between the phases in which two interfaces are present; upon crossing this segment discontinuity of $\theta_{2}$ is observed. It is worthwhile to note that the line B-E corresponds - for the cone geometry considered in this paper - to the phase transition found by Widom [10] when analyzing a liquid drop sessile on a flat substrate. The difference between the phases separated by the line B-E becomes smaller and smaller upon approaching point $\mathrm{B}$ and finally disappears at this point. To examine the shape of the B-E line in the neighborhood of this point we look for approximate analytical solution of (17). For $\bar{\eta} \rightarrow 0$ this set of equations can be solved perturbatively and one finds that

$$
\left(\pi-\theta_{0}\right) \sim \bar{\eta}^{1 / 2} .
$$

The G-F-A-D line is the line of transitions between configurations with one and with two interfaces, respectively. Along the segment G-F-A this transition is continuous while 
along the segment A-D it is first-order. It corresponds to discontinuity of the value of the contact angle $\theta_{2}$ upon crossing the segment A-C one additionally observes the discontinuity of $\theta_{1}$. Thus point $\mathrm{A}\left(\eta=0, \theta_{0}=\pi / 2+\psi\right)$, which played a distinguished role on figure 2, tends out to be the tricritical point in the enlarged space of system parameters. Analyzing the shape of the A-D line in the vicinity of the tricritical point leads again to the conclusion that

$$
\left(\theta_{0}-\left(\frac{\pi}{2}+\psi\right)\right) \sim \bar{\eta}^{1 / 2} .
$$

One also observes that upon approaching the tricritical point the discontinuity of $\theta_{2}$ decreases to zero as the square root of $\bar{\eta}$.

As far as the dependence on the cone opening angle $\psi$ is considered one observes the decrease of the region $\mathrm{BACD}$ on figure 3 upon increasing $\psi$ towards $\pi / 2$; in this limit points $\mathrm{A}$ and $\mathrm{D}$ tend towards point $\mathrm{B}$. This area corresponds to situations in which positive line tension and the values of $\theta_{0}$ close to $\pi$ stabilize the configurations with two interfaces. Finally, for $\psi=\pi / 2$ one recovers the phase diagram discussed by Widom [10].

\section{Conclusions}

In these paper we have investigated the configurations of a non-volatile liquid drop placed in a cone. Our macroscopic analysis based on the constrained free energy leads to the phase diagram parameterized by the Young angle $\theta_{0}$ and the dimensionless line tension $\bar{\eta}$. The interesting feature of the phase diagram is the existence of lines of the first- and the second-order transitions corresponding either to discontinuous or continuous changes of the contact angles. One also finds the tricritical point and the parabolic shape of the transition line in its vicinity. Another property of the system is that the negative line tension stabilizes the so-called wetting configuration into the range of non-zero values of the Young angle. This wetting configuration is characterized by the presence of a single interface and zero contact angle.

One should keep in mind that the present analysis is restricted to interfacial configurations which are cylindrically symmetric and smooth at the cone axis. In the case of negative line tension with large absolute values one might expect still a different class of equilibrium configuration which consist of separate "rings" extending along the cone. Analogous situations appear also in the analysis of a sessile drop on flat substrate; this will be the subject of our future analysis.

\section{Acknowledgment}

The authors gratefully acknowledge the discussions with prof. Hans W. Diehl and prof. Herbert Wagner, and the support by the Foundation for German-Polish Collaboration under Grant. No. 3269/97/LN. 


\section{References}

[1] Widom B 1973 Surface Tension of Fluids Phase Transitions in Critical Phenomena vol 2, ed by C Domb and J L Lebowitz (London: Academic)

[2] de Gennes P. G. 1985 Rev. Mod. Phys. 57827

[3] Dietrich S 1988 Wetting phenomena Phase Transitions and Critical Phenomena vol 12, ed C Domb $\mathrm{C}$ and J L Lebowitz (London: Academic) p 1

[4] Rowlinson J S and Widom B 1989 Molecular Theory of Capilarity (Oxford: Clarendon Press)

[5] Schick M 1990 in Liquids at Interfaces. Proceedings of the Los Houches Summer School in Theoretical Physics. Session XLVIII, edited by Chavrolin J, Joanny J F and Zinn-Justin J (Amsterdam: North-Holland)

[6] Indekeu J O 1994 International Journal of Modern Physics B 830

[7] Boruvka L and Neumann A W 1977 J. Chem. Phys. 665464.

[8] Widom B 1990 Proc. of The Gibbs Symp. (Yale) (New York: American Math. Soc.) p 73

[9] van Giessen A E, Bukman D J and Widom B 1999 Mol. Phys. 961335

[10] Widom B 1995 J. Phys. Chem. 992803.

[11] Concus P and Finn R 1998 Phys. Fluids 1039

[12] Rejmer K, Dietrich S, and Napiórkowski M 1999 Phys. Rev. E 604027.

[13] Minimalization was done by means of Secant-type Unconstrained Minimization problem SoLver (SUMSL) from a set of subroutines designed by David M. Gay; Gay D M 1983 ACM Transactions on Mathematical Software 9503.

[14] Li D 1996 Coll. Surf. 1161

Drelich J 1996 Coll. Surf. 11643

[15] Grigoriev I S, Meilikhov E Z and Radzig A A, editors 1997 Handbook of Physical Quantities (Boca Raton - New York - London - Tokyo: CRC Press) 